\title{
CULTIVO DE Chlorella fusca LEB 111 E Chlorella minutissima LEB 114 ISOLADAS DE LAGOAS DE TRATAMENTO DE EFLUENTES DE ORIGEM TERMELÉTRICA
}

\author{
B. S. VAZ ${ }^{1}$, J. H. DUARTE ${ }^{1}$, E. G. MORAIS ${ }^{1}$, M. G. MORAIS ${ }^{1}$ e J. A. V. $\operatorname{COSTA}^{1}$ \\ ${ }^{1}$ Universidade Federal do Rio Grande, Escola de Química e Alimentos, Laboratório de Engenharia \\ Bioquímica \\ E-mail para contato: jorgealbertovc@gmail.com
}

\begin{abstract}
RESUMO - $\mathrm{O} \mathrm{CO}_{2}$ é o principal gás de efeito estufa e sua concentração na atmosfera tem aumentado rapidamente desde o início da industrialização. O objetivo deste estudo foi avaliar parâmetros cinéticos de crescimento de Chlorella fusca LEB-111 e Chlorella minutissima LEB-114, cultivadas utilizando $\mathrm{CO}_{2}$ como fonte de carbono. As microalgas foram cultivadas em meio BG-11 (Rippka et al., 1979) e BG-11 com substituição da fonte de carbono por $10 \%(\mathrm{v} / \mathrm{v})$ de $\mathrm{CO}_{2}$. Os maiores valores de crescimento celular máximo e produtividade máxima foram obtidos quando as microalgas Chlorella fusca LEB 111 e Chlorella minutissima LEB 114 foram cultivadas substituindo-se a fonte de carbono por $10 \%$ de $\mathrm{CO}_{2}(\mathrm{v} / \mathrm{v})\left(0,91,0,94 \mathrm{~g} . \mathrm{L}^{-1}, 0,08 \mathrm{~g} \cdot \mathrm{L}^{-1} \cdot \mathrm{d}^{-1}\right.$ e $0,08 \mathrm{~g} \cdot \mathrm{L}^{-1} \cdot \mathrm{d}^{-1}$, respectivamente). Além de minimizar os problemas ambientais, o uso de fontes alternativas como $\mathrm{CO}_{2}$ reduz os custos com esse nutriente, que representa grande parte dos gastos de produção de tais micro-organismos.
\end{abstract}

\section{INTRODUÇÃO}

Os atuais níveis de dióxido de carbono $\left(\mathrm{CO}_{2}\right)$ na atmosfera são considerados a principal causa da mudança climática, uma questão de grande preocupação mundial (García et al., 2007). O aquecimento global, induzido pelo aumento das concentrações dos gases de efeito estufa na atmosfera, pode causar impactos desastrosos na natureza. Estes impactos incluem a redução da produção de alimentos, extinção de espécies, alteração no suprimento de água doce, maior número de ciclones, secas, tempestades e enchentes. O dióxido de carbono $\left(\mathrm{CO}_{2}\right)$ é o principal gás de efeito estufa e suas concentrações têm aumentado rapidamente desde o início da industrialização. Em 1997, 7,4 bilhões de toneladas de $\mathrm{CO}_{2}$ foram liberados para a atmosfera a partir de fontes antropogênicas, e até o ano de 2100, este número tende a aumentar para 26 bilhões de toneladas (Chiu et al., 2008; Costa e Morais, 2011). 


\section{9 a 22 de outubro de 2014 \\ Florianópolis/SC}

Diversas alternativas têm sido estudadas a fim de promover a redução das emissões de termelétricas a carvão mineral, como tratamentos físicos e químicos que podem ser utilizados por meio do emprego de fontes de energia renováveis ou de sequestro de carbono (Chiu et al., 2009). Uma alternativa para redução de $\mathrm{CO}_{2}$ sem alteração na matriz energética mundial é o cultivo de microalgas. A fonte de carbono necessária para o cultivo de microalgas representa cerca de $60 \%$ dos custos com nutrientes. As exigências nutricionais para o cultivo desses micro-organismos podem estar disponíveis em resíduos industriais, transformando o que é considerado um problema em matériaprima para a obtenção de produtos com alto valor agregado (Costa e Morais, 2011).

As microalgas são responsáveis por mais de $50 \%$ do $\mathrm{CO}_{2}$ fixado no planeta. As espécies do gênero Chlorella são importantes componentes do fotoplâncton de água doce e estima-se que elas fixam mais de 1 bilhão de toneladas de $\mathrm{CO}_{2}$ nos oceanos e nas águas doces a cada ano (Margulis e Schwartz, 2001). A capacidade fotoautotrófica das microalgas converte $\mathrm{CO}_{2} \mathrm{em}$ biomassa, onde além de minimizar os problemas ambientais, o uso de fontes alternativas de nutrientes como o $\mathrm{CO}_{2}$ reduz os custos do processo (Chiu et al., 2008; Chiu et al., 2009; Rosa et al., 2011). Com isso, as microalgas têm sido muito investigadas para biofixação do $\mathrm{CO}_{2}$ da atmosfera, contribuindo assim com redução do efeito estufa e consequente aquecimento global.

Alguns pesquisadores relatam que espécies de microalgas nativas podem ser mais tolerantes a condições locais, apresentando maiores taxas fotossintéticas (Radmann et al., 2011; Salih, 2011). O isolamento de microalgas provenientes da região pode maximizar a eficiência destes microorganismos em biofixar $\mathrm{CO}_{2}$. Dessa forma, o objetivo deste estudo foi avaliar parâmetros cinéticos de crescimento de Chlorella fusca LEB 111 e Chlorella minutissima LEB 114, cultivadas utilizando $\mathrm{CO}_{2}$ como fonte de carbono.

\section{MATERIAIS E MÉTODOS}

\subsection{Micro-organismos e meio de cultivo}

As microalgas Chlorella fusca LEB 111 e Chlorella minutissima LEB 114 isoladas da lagoa de tratamento de efluentes da Usina Termelétrica Presidente Médici - UTPM (latitude 2436' '13''S e longitude $52^{\circ} 32^{\prime} 43^{\prime \prime} \mathrm{W}$ ) (Candiota - RS) foram utilizadas neste estudo. O meio de cultivo utilizado foi BG-11 (Rippka et al., 1979) e BG-11 modificado, onde a fonte de carbono padrão do meio BG-11 $\left(\mathrm{Na}_{2} \mathrm{CO}_{3}\right)$ foi substituída por $\mathrm{CO}_{2}$ comercial.

\subsection{Condições de cultivo}

Os cultivos foram realizados em fotobiorreatores fechados de $2 \mathrm{~L}$, conforme mostra a Figura 1 . A temperatura foi mantida à $30^{\circ} \mathrm{C}$ em câmara termostatizada com fotoperíodo de $12 \mathrm{~h}$ claro/escuro (Reichert et al., 2006) e 43,2 $\mu \mathrm{mol} \mathrm{m} \mathrm{m}^{-2} \mathrm{~s}^{-1}$ de iluminância fornecidos por lâmpadas fluorescentes (General Eletric). A aeração foi fornecida por ar comprimido misturado ao gás disposto em cilindro industrial (White Martins, Brasil) com vazão de $0,05 \mathrm{vvm}$ contendo $10 \%$ (v/v) de $\mathrm{CO}_{2}$. O gás foi adicionado aos cultivos por 10 min a cada $2 \mathrm{~h}$ durante o período claro. Os experimentos foram realizados em duplicata. 


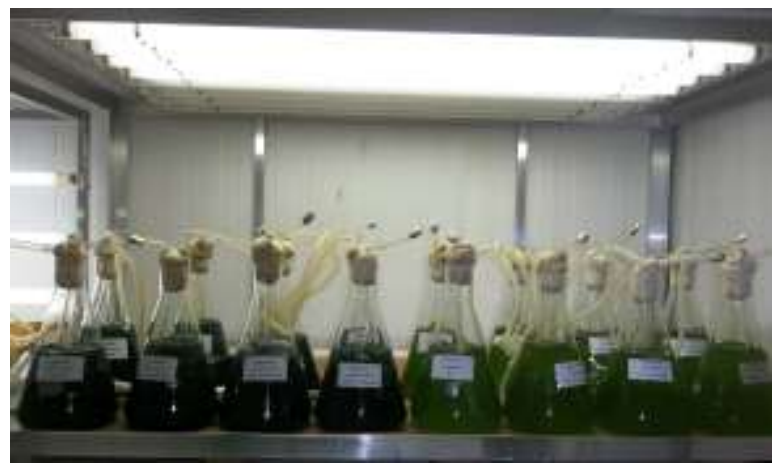

Figura 1 - Cultivos realizados em fotobiorreatores do tipo erlenmeyer de $2 \mathrm{~L}$.

\subsection{Determinações analíticas}

A concentração de biomassa monitorada diariamente foi determinada através da leitura da densidade ótica das culturas a $670 \mathrm{~nm}$ (Rosa et al., 2011) em espectrofotômetro (QUIMIS Q798DRM), com curva de calibração que relaciona densidade ótica com peso seco de biomassa para cada microalga (Radmann et al., 2007). O pH dos cultivos foi determinado a cada 24 h com pHmetro digital (LUTRON PH-221).

\subsection{Parâmetros cinéticos}

Os valores de concentração de biomassa foram utilizados para determinar as velocidades específicas máximas de crescimento $\left(\mu_{\text {máx }}, \mathrm{d}^{-1}\right)$, concentrações celulares máximas $\left(\mathrm{X}_{\text {máx }}, \mathrm{g}\right.$. $\left.\mathrm{L}^{-1}\right)$ e produtividades máximas $\left(\mathrm{P}_{\text {máx }}, \mathrm{g} . \mathrm{L}^{-1} \cdot \mathrm{d}^{-1}\right)$. A produtividade máxima foi obtida segundo a Equação 1, onde $\mathrm{X}_{\mathrm{t}}$ é a concentração de biomassa $\left(\mathrm{g}\right.$. $\left.\mathrm{L}^{-1}\right)$ no tempo $\mathrm{t}(\mathrm{d})$, e $\mathrm{X}_{0}$ a concentração de biomassa $\left(\mathrm{g}\right.$. $\mathrm{L}^{-1}$ ) no tempo $t_{0}(\mathrm{~d})$ (Schmidell et al., 2001). A velocidade específica máxima de crescimento $\left(\mu_{\text {máx }}, \mathrm{d}^{-1}\right)$ foi obtida por regressão exponencial aplicada à fase logarítmica, segundo a Equação 2 (Bailey e Ollis, 1986).

$$
\begin{aligned}
P & =\frac{X_{t}-X_{0}}{t-t_{0}} \\
\mu & =\frac{1}{X} \frac{d X}{d t}
\end{aligned}
$$

\subsection{Análise estatística}

Os resultados foram avaliados através de Análise de Variância (ANOVA) e teste de Tukey para comparação das médias dos parâmetros cinéticos, com nível de significância de $95 \%(\mathrm{p} \leq 0,05)$. 


\section{RESULTADOS E DISCUSSÃO}

A Figura 2 apresenta as curvas de crescimento das microalgas Chlorella fusca LEB 111 e Chlorella minutissima LEB 114 cultivadas com meio BG-11 padrão e BG-11 modificado com 10\% de $\mathrm{CO}_{2}$ como fonte de carbono. Os valores médios dos parâmetros cinéticos de crescimento das microalgas são apresentados na Tabela 1 .

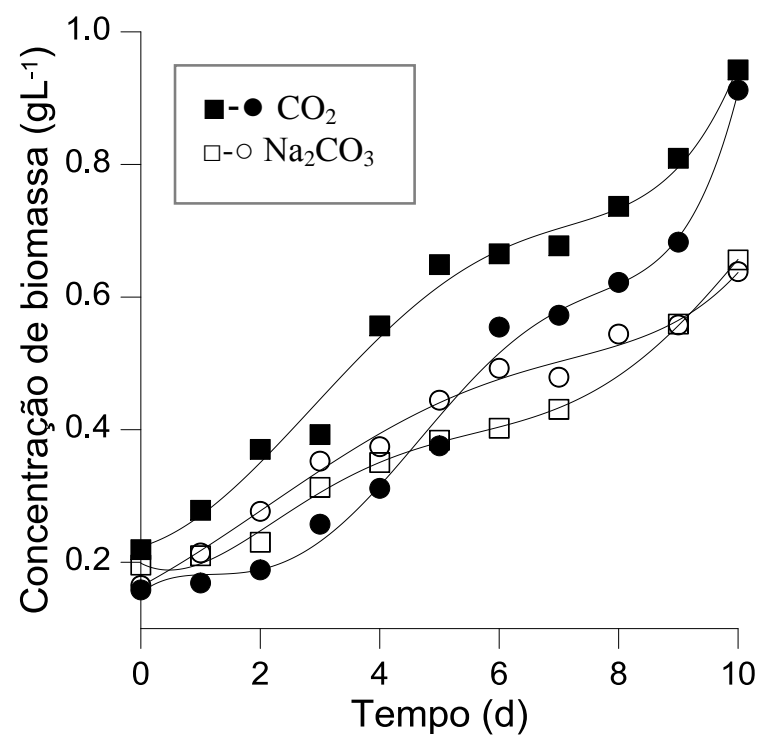

Figura 2 - Curvas de crescimento das microalgas Chlorella fusca LEB 111(•-०) e Chlorella minutissima LEB 114 ( $\square-\square)$ cultivadas em diferentes fontes de carbono.

Os maiores valores de concentração de biomassa máxima, 0,94 e 0,91 g. $\mathrm{L}^{-1}$, foram obtidos nos cultivos das microalgas Chlorella minutissima LEB 114 e Chlorella fusca LEB 111 com 10\% de $\mathrm{CO}_{2}$, respectivamente. Nos experimentos com a fonte de carbono padrão do meio BG-11 $\left(\mathrm{Na}_{2} \mathrm{CO}_{3}\right)$, os valores de crescimento celular máximo diferiram estatisticamente dos ensaios com $\mathrm{CO}_{2}$. As máximas produtividades $\left(0,08 \mathrm{~g} \cdot \mathrm{L}^{-1} \cdot \mathrm{d}^{-1}\right)$ também foram obtidas nos cultivos das microalgas com $\mathrm{CO}_{2}$, não havendo diferença estatística significativa $(\mathrm{p}<0,05)$ dos demais experimentos (Tabela 1$)$.

Tabela 1 - Concentração celular máxima $\left(\mathrm{X}_{\text {máx }}\right)$, velocidade específica máxima de crescimento $\left(\mu_{\text {máx }}\right)$ e produtividade máxima $\left(\mathrm{P}_{\text {máx }}\right)$ obtidos para as microalgas Chlorella fusca e Chlorella minutíssima.

\begin{tabular}{|c|c|c|c|c|}
\hline Microalga & $\begin{array}{c}\text { Fonte de } \\
\text { carbono }\end{array}$ & $\mathrm{X}_{\text {máx }}\left(\mathrm{g} \cdot \mathrm{L}^{-1}\right)$ & $\mathrm{P}_{\text {máx }}\left(\mathrm{g} \cdot \mathrm{L}^{-1} \cdot \mathrm{d}^{-1}\right)$ & $\mu_{\text {máx }}\left(\mathrm{d}^{-1}\right)$ \\
\hline \multirow{2}{*}{ Chlorella fusca LEB 111 } & $\mathrm{Na}_{2} \mathrm{CO}_{3}$ & $0,64 \pm 0,01^{\mathrm{a}}$ & $0,06 \pm 000^{\mathrm{a}}$ & $0,07 \pm 0,02^{\mathrm{c}}$ \\
\cline { 2 - 5 } & $\mathrm{CO}_{2}$ & $0,91 \pm 0,07^{\mathrm{b}}$ & $0,08 \pm 0,01^{\mathrm{a}}$ & $0,24 \pm 000^{\mathrm{b}}$ \\
\hline \multirow{2}{*}{ Chlorella minutissima LEB 114 } & $\mathrm{Na}_{2} \mathrm{CO}_{3}$ & $0,65 \pm 0,08^{\mathrm{a}}$ & $0,05 \pm 0,01^{\mathrm{a}}$ & $0,20 \pm 0,01^{\mathrm{ab}}$ \\
\cline { 2 - 5 } & $\mathrm{CO}_{2}$ & $0,94 \pm 0,08^{\mathrm{b}}$ & $0,08 \pm 0,02^{\mathrm{a}}$ & $0,18 \pm 0,01^{\mathrm{a}}$ \\
\hline
\end{tabular}

Letras iguais na mesma coluna as médias não diferem significativamente $(\mathrm{p}<0,05)$ pelo Teste de Tukey. 


\section{9 a 22 de outubro de 2014 \\ Florianópolis/SC}

A partir da Figura 1 é possível observar que o experimento que obteve maior valor de concentração de biomassa celular $\left(0,94 \mathrm{~g}\right.$. $\left.\mathrm{L}^{-1}\right)$ foi o que apresentou crescimento celular desde a inoculação, em taxa aproximadamente constante, não havendo a fase "lag" ou de adaptação. A fase "lag" ocorre após a inoculação da microalga no meio de cultivo, não havendo reprodução, pois as células estão sintetizando enzimas necessárias para o crescimento em diferentes condições que o inóculo inicial foi mantido. A duração dessa fase depende da concentração inicial do inóculo, do tempo de pré-cultivo e do estado fisiológico do micro-organismo (Schmidell et al., 2001).

$\mathrm{O}$ pH dos cultivos manteve-se entre 8,7 e 12,5. Microalgas do gênero Chlorella mantem crescimento em pH entre 6,5 e 7,5. As cepas de Chlorella fusca LEB 111 e Chlorella minutissima LEB 114 por serem isoladas de lagoas de tratamento de efluentes de origem termelétrica $(\mathrm{pH} \mathrm{9,8)} \mathrm{são}$ resistentes à alcalinidade. Em cultivos de microalgas geralmente é necessária uma fonte externa de carbono que participe do equilíbrio químico $\mathrm{CO}_{2} \leftrightarrow \mathrm{HCO}_{3}{ }^{-} \leftrightarrow \mathrm{CO}_{3}{ }^{2-} . \mathrm{O} \mathrm{CO}_{2}$ é a fonte de carbono para o crescimento fotossintético e autotrófico de microalgas. As microalgas utilizam a fotossíntese como metabolismo principal para a obtenção de carbono orgânico através do carbono inorgânico contido no $\mathrm{CO}_{2}$ (Costa et al., 2003).

O íon bicarbonato entra na célula por transporte ativo e a enzima anidrase carbônica age sobre o bicarbonato liberando o $\mathrm{CO}_{2}$, que é dissociado em bicarbonato $\left(\mathrm{HCO}_{3}{ }^{-}\right)$e íons $\mathrm{H}^{+}$, e o bicarbonato pode ser dissociado em $\mathrm{CO}_{2}$ ou íons carbonato $\left(\mathrm{CO}_{3}{ }^{-2}\right)$ dependendo do $\mathrm{pH}$ do meio de cultivo (Basu, 2013; Binaghi et al., 2003). O pH determina a distribuição das espécies químicas. Quanto maior o pH do meio, mais facilmente o $\mathrm{CO}_{2}$ esta disponível no meio e é convertido em $\mathrm{CO}_{3}{ }^{2-}$ (Radmann et al., 2011). $\mathrm{O} \mathrm{CO}_{2}$ é a fonte de carbono para o crescimento fotossintético e autotrófico de microalgas.

Valores máximos de concentração de biomassa e consequente máxima produtividade obtidos para as microalgas $C$. fusca e $C$. minutissima podem ter sido devido ao fato de serem espécies isoladas de lagoas de tratamento de efluentes próximas as emissões de termelétricas. Alguns pesquisadores acreditam que espécies de microalgas nativas sejam mais tolerantes a condições locais, apresentando maiores taxas de fotossíntese e produção de biomassa. $\mathrm{O}$ isolamento de microalgas provenientes da região é, dessa forma, uma alternativa para maximizar a eficiência das microalgas em biofixar $\mathrm{CO}_{2}$. Assim, diminuiria o risco de impacto ambiental, a dependência de importar cepas seria eliminada e, haveria tolerância a altas concentrações dos gases de combustão emitidos em usinas termelétricas (Brown et al., 1996; Radmann et al., 2011; Salih, 2011).

Morais e Costa (2007) isolaram as microalgas Scenedesmus obliquus e Chlorella kessleri, de lagoas de tratamento de efluentes da Usina Termelétrica Presidente Médici (Candiota- RS), e verificaram que estas apresentaram crescimento quando cultivadas com $18 \%$ de $\mathrm{CO}_{2}$. Lee et al. (2002) e Rosa et al. (2011) relataram que concentrações acima de $12 \%$ e $18 \%$ de $\mathrm{CO}_{2}$ são toleradas pelas microalgas. Foram obtidos $2,0 \mathrm{~g}$. L $\mathrm{L}^{-1}$ para a microalga Chlorella sp. cultivada com $15 \%$ de $\mathrm{CO}_{2}$. A Chlorella tem sido estudada como método alternativo de absorção de $\mathrm{CO}_{2}$ atmosférico, possuindo alta capacidade de fixação de $\mathrm{CO}_{2}$ e resistência quando exposta a concentrações deste gás superiores ao emitido em usinas termelétricas (Morais e Costa, 2007). 


\section{CONCLUSÃO}

Os maiores valores de concentração de biomassa celular máxima e produtividade máxima foram obtidos nos cultivos das microalgas com $\mathrm{CO}_{2}$ como fonte de carbono. A utilização de gases industriais torna-se alternativa em culturas de microalgas, visto que a principal barreira para o cultivo industrial é o custo com os nutrientes, principalmente a fonte de carbono. Nesse sentido, tecnologias baseadas em algas podem reduzir as emissões de gases de efeito estufa de usinas termelétricas, além de reduzir os custos com nutrientes necessários ao seu crescimento.

\section{REFERÊNCIAS}

BAILEY, J. E.; OLLIS, D. F. Biochemical Engineering Fundamentals. $2^{\mathrm{a}}$ ed. Singapore: McGrawHill, 1986.

BASU, S.; ROY, A. S.; MOHANTY, K.; GHOSHAL, A. K. Enhanced $\mathrm{CO}_{2}$ sequestration by a novel microalga: Scenedesmus obliquus SA1 isolated from bio-diversity hotspot region of Assam, India. Bioresour. Technol., v. 143, p. 369-377, 2013.

BINAGHI, L.; BORGHI, A. D.; LODI, A.; CONVERTI, A.; BORGHI, M. D. Batch and fed-batch uptake of carbon dioxide by Spirulina platensis. Process Biochem., v. 38, p. 1341-1346, 2003.

BROWN, L.M. Uptake of carbon dioxide from flue gas by microalgae. Energy Convers. Manage.,, 37, 1363 - 1367, 1996.

CHIU, S. Y.; KAO, C. Y.; CHEN, C. H.; KUAN, T. C.; ONG, S. C.; LIN, C. S. Reduction of CO 2 by a high-density culture of Chlorella sp. in a semicontinuous photobioreactor. Bioresour. Technol., v. 99, p. 3389-3396, 2008.

CHIU, S. Y.; KAO, C. Y.; TSAI, M, T.; ONG, S. C.; CHEN, C. H.; LIN, C. S. Lipid accumulation and $\mathrm{CO}_{2}$ utilization of Nannochloropsis oculata in response to $\mathrm{CO}_{2}$ aeration. Bioresour. Technol., v. 100, p. 833-838, 2009.

COSTA, J. A.V.; COLLA, L. M.; DUARTE FILHO, P. Spirulina platensis growth in open raceway ponds using fresh water supplemented with carbono, nitrogen and metal íons. Z Naturforsch. v. 58, p. 76-80, 2003.

COSTA, J. A. V.; MORAIS, M. G. The role of biochemical engineering in the production of biofuels from microalgae. Bioresour. Technol., v. 102, n. 1, p.2-9, 2011.

GARCÍA, L. M.; GARCÍA, A. I.; MORÁN, A. Isolation and selection of microalgae species for the $\mathrm{CO}_{2}$ bio-fixation. J. Biotechnol., 131S, p. S122-S126, 2007.

LEE, J. S.; KIM, D. K.; LEE, J. P.; PARK, S. C; KOH, J. H; CHO, H. S; KIM, S. W. Effects of $\mathrm{SO}_{2}$ and NO on growth of Chlorella sp. KR-1. Bioresour. Technol., v. 82, p. 1-4, 2002.

MARGULIS, L.; SCHWARTZ, K. V. Cinco Reinos: Um Guia Ilustrado dos Filos da Vida na Terra.

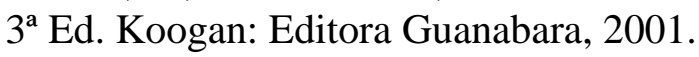


MORAIS, M. G.; COSTA, J. A. V. Isolation and selection of microalgae from coal fired thermoelectric power plant for biofixation of carbono dioxide. Energy Convers. Manage., v. 48, p. 2169-2173, 2007.

RADMANN, E. M.; REINEHR, C. O.; COSTA, J. A. V. Optimization of the repeated batch cultivation of microalga Spirulina platensis in open raceway ponds. Aquaculture, v. 265, p. 118126, 2007.

RADMANN, E. M.; CAMERINI, F. V.; SANTOS, T. D.; COSTA, J. A. V. Isolation and application of $\mathrm{SO}_{\mathrm{X}}$ and $\mathrm{NO}_{\mathrm{X}}$ resistant microalgae in Biofixation of $\mathrm{CO}_{2}$ from thermoelectricity plants. Energy Convers. Manage., v. 52, p. 3132-3136, 2011.

REICHERT, C. C.; REINEHR, C. O.; COSTA, J. A. V. Semicontinuous cultivation of the cyanobacterium Spirulina platensis in a closed photobioreactor. Braz J Chem Eng., v. 23, p. 2328, 2006.

RIPPKA, R.; DERUELLES, J.; WATERBURY, J. W.; HERDMAN, M.; STANIER, R. G. Genetic assignments, strain histories and properties of pure cultures of Cyanobacteria. J. Gen. Microbiol., v. 111, p. 1-61, 1979.

ROSA, A. P. C.; CARVALHO, L. F.; GOLDBECK, L.; COSTA, J. A. V. Carbon dioxide fixation by microalgae cultivated in open bioreactors. Energy Convers. Manage., v. 52, p. 3071-3073, 2011.

SALIH, F. M. Microalgae Tolerance to High Concentrations of Carbon Dioxide: A Review. J. Environ. Prot., v. 2, p. 648-654, 2011.

SCHMIDELL, W.; LIMA, A. U.; AQUARONE, E.; BORZANI, W. Biotecnologia Industrial. $2^{\mathrm{a}}$ Ed. São Paulo: Edgard Blücher LTDA, 2001. 